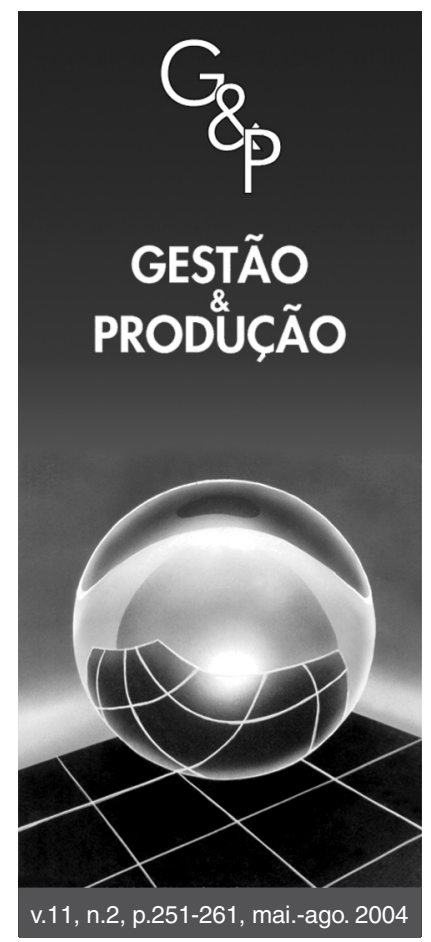

\title{
REPRESENTAÇÃO EM ESPAÇO DE ESTADOS PARA A FLEXIBILIDADE DE ROTEAMENTO
}

\author{
Leonardo Rosa Rohde \\ Faculdade de Administração, Contabilidade e Economia, \\ Pontifícia Universidade Católica do Rio Grande do Sul, \\ Av. Ipiranga, 6681 (prédio 50), CEP 90619-900, Porto Alegre, RS, \\ e-mail: Irrohde@pucrs.br \\ Denis Borenstein \\ Escola de Administração, \\ Universidade Federal do Rio Grande do Sul, \\ Rua Washington Luiz, 855, CEP 90010-460, Porto Alegre, RS, \\ e-mail: denisb @ea.ufrgs.br
}

\section{Resumo}

Este artigo descreve uma representação em espaço de estados para a flexibilidade de roteamento em sistemas de manufatura. A flexibilidade de roteamento é representada em três níveis de detalhamento, como segue: (i) grafo de precedências; (ii) grafo de transição de estados para a flexibilidade de seqüência; e (iii) grafo de transição de estados para a flexibilidade de roteamento. A terceira representação é a mais completa, sendo capaz de enumerar todas as possíveis rotas de uma peça no sistema de manufatura. Neste artigo ainda, será abordada a relação entre a representatividade e complexidade computacional para cada representação, auxiliando o processo de seleção do nível de representação mais adequado a um determinado contexto. A versatilidade desta representação permite que esta seja facilmente vinculada a outras ferramentas de análise e apoio, para o controle e projeto de sistemas flexíveis de manufatura, permitindo que estes sejam devidamente avaliados, compreendidos e mensurados. Por fim, a eficácia da representação é demonstrada por meio de sua aplicação em problemas de roteamento e mensuração da flexibilidade em sistemas de manufatura.

Palavras-chave: flexibilidade de roteamento, representação em espaço de estados, projeto de manufaturas.

\section{Introdução}

A flexibilidade é um dos conceitos chave na manufatura contemporânea - um importante atributo dos sistemas de manufatura, que permite às companhias tornarem-se competitivas em um ambiente dinâmico (Kochikar e Narendran, 1992). A flexibilidade é o atributo que distingue FMSs (do inglês, flexible manufacturing system) dos tradicionais processos de produção em massa, como por exemplo, os sistemas automatizados de produção em linha. A flexibilidade é definida por alguns autores como "a capacidade de um sistema de manufatura de lidar, efetivamente, com mudanças internas ou externas" (Gupta e Buzacott, 1989). Por causa do grande número de tipos de mudanças (quebra de máquinas, mudança no volume de produção, etc.) é muito difícil encontrar um conceito único de flexibilidade (Azzone e Bertelè, 1991). Conseqüentemente, é necessário dividir o conceito de flexibilidade em conceitos elementares, que estão associados a certos tipos de elementos e distúrbios de um sistema de manufatura.

Em um ambiente de manufatura dedicada, cada peça está associada a um simples processo, consistindo em uma sequiência fixa e pré ordenada de operações (Benjaafar e Ramakrishnam, 1996). Hutchinson e Pflughoeft (1994) chamam esta situação de TTP (do inglês, traditional process plans). Quando consideramos um sistema de manufatura tradicional, existe pouca oportunidade de melhoria das decisões de alocação de recursos, face aos possíveis distúrbios ou alterações das condições do sistema. Como consequiência, o planejamento offline deve ser cegamente seguido. Esta situação é facilmente revertida, quando consideramos um FMS. Tais sistemas são capazes de realizar operações numa seqüência não prefixada e processadas por diferentes máquinas. Assim, existe a oportunidade de mudar dinamicamente os planos de manufatura, aumentando a possibilidade de decisões de alocações de recursos frente aos distúrbios ou mudanças no ambiente. 
Existem três tipos de flexibilidade associadas à manufatura de uma peça: flexibilidade de seqüência, flexibilidade de máquinas e flexibilidade de roteamento (Benjaafar e Ramakrishnam, 1996; Hutchinson e Pflughoeft, 1994; Sethi e Sethi, 1990). Flexibilidade de sequiência refere-se à possibilidade de alternar a sequiência de operações em uma peça, levando em consideração as restrições e especificações de seu projeto. Flexibilidade de máquinas relaciona-se à possibilidade de uma operação poder ser executada em mais de uma máquina. Flexibilidade de roteamento refere-se à capacidade do sistema de manufatura de permitir, simultaneamente, a flexibilidade de máquinas e sequiência. Diversos pesquisadores têm demonstrado o potencial de melhoria na performance de manufaturas sob condições de flexibilidade (Benjaafar, 1994; Hancock, 1989; Hutchinson e Pflughoeft, 1994). Uma vez que a flexibilidade pode ser um importante atributo no que tange a melhoria dos sistemas, o desenvolvimento de modelos e algoritmos capazes de representarem a flexibilidade é um importante passo para o projeto, avaliação e controle destes sistemas. Além disso, o desenvolvimento de representações para a flexibilidade de roteamento permitiria um importante passo para melhor entender as conseqüências da introdução da flexibilidade na manufatura, em virtude dos altos investimentos associados.

Diversas metodologias para representação da flexibilidade de roteamento têm sido utilizadas. Lin e Solberg (1991) desenvolveram um dígrafo AND/OR para representar a flexibilidade de seqüência. Nesta representação, as operações e restrições de uma peça estão associadas aos nós e arcos do grafo, respectivamente. Wei e Egbelu (2000) usaram a mesma abordagem para representar os processos de manufatura, levando em consideração os projetos e informações de cada produto. Infelizmente, este tipo de representação pode gerar processos de produção, para os quais não existem máquinas no sistema (Benjaafar, 1994). Yang, Qiao e Jiang (1998), Borenstein (2000), e Benjaafar e Ramakrishnam (1996) propuseram representações baseadas em dígrafos OR (ou árvores), em que as operações são representadas por nós e as restrições de precedência, por arcos. Kochikar e Narendram (1992) usaram o formalismo de transição de estados das redes de Petri, para representar todas as possibilidades de estados de uma peça. Entretanto, todas as representações acima citadas são impraticáveis por causa do considerável esforço computacional necessário para armazená-las. As representações crescem rapidamente na medida em que se aumenta a complexidade dos planos de produção, como consequiência das múltiplas representações de uma mesma operação para diferentes seqüências.

Apesar da diversidade de representações descritas na literatura, os autores desconhecem modelos na literatura sobre flexibilidade de roteamento que compreendam, simultaneamente, os seguintes aspectos: (i) capacidade de gerar todos os possíveis roteamentos de manufatura; e (ii) desenvolvimento de um algoritmo completo e correto que gere a representação eficientemente.
O principal objetivo deste trabalho é o desenvolvimento de tal modelo. Este artigo apresenta uma representação compacta em espaço de estados, para todas as possíveis rotas de manufatura para uma determinada peça. A representação por espaço de estados é uma das técnicas mais apropriadas para analisar sistemas discretos e dinâmicos (Kochikar e Narendran, 1992). Nesta abordagem, um sistema é representado pelos estados correspondentes às possíveis situações que podem existir na manufatura. A idéia por trás desta representação é selecionar um mínimo de atributos (chamados de variáveis de estado), reduzindo o espaço de estados, porém mantendo o comportamento e as relações de interesse. O principal objetivo é obter um modelo em dimensão reduzida que se aproxime do comportamento original do sistema (Hwang, Guo e Shieh, 1991). Para representarmos a flexibilidade de roteamento, utilizamos diferentes níveis de representação. Limites computacionais são apresentados para cada nível de representação, para facilitar ao usuário a seleção da melhor representação.

A principal contribuição deste artigo é o desenvolvimento de diferentes representações capazes de enumerar, se necessário, todas as possíveis seqüências e roteamentos de uma peça. Estes níveis de representações podem ser vinculados a métodos de avaliação e análise de FMSs. Uma vantagem adicional do modelo é que ele pode ser aplicado a qualquer estágio de um sistema de manufatura - mesmo para uma análise em tempo real. Estes benefícios são obtidos a partir da concepção do modelo, que fornece uma clara e teórica representação da flexibilidade de roteamento. Em resumo, tal representação fornece a oportunidade para aumentar a flexibilidade com a qual os produtos são manufaturados, e permite uma escolha dinâmica e inteligente das rotas de manufatura, baseada em um conjunto de critérios definidos pelo engenheiro de manufatura.

\section{Representação em espaço de estados para a flexibilidade de roteamento}

O modelo desenvolvido para a flexibilidade de roteamento é composto por três representações com diferentes níveis de detalhamento: (i) grafo de precedências; (ii) grafo de transição de estados para a flexibilidade de sequiência; e (iii) grafo de transição de estados para a flexibilidade de roteamento. Estas representações são obtidas pela projeção do espaço de estados completo de um sistema de manufatura. Projeção é uma técnica de redução do espaço de estados, baseada na agregação de estados, seguindo critérios predefinidos, de forma a preservar certos aspectos comportamentais do sistema, enquanto ignora outros (Morgan e Razouk, 1987). O objetivo é reduzir o completo espaço de estados de um sistema de manufatura, um clássico problema nãodeterminístico polinomial (classe NP) (Kochikar e Narendran, 1992), em um outro espaço mais simples e tratável. A primeira representação leva em consideração apenas os aspectos de descrição das operações e suas precedências; a 
segunda representação considera como as operações de manufatura podem ser seqüenciadas; e a terceira representação adiciona máquinas ao modelo, representando as possíveis rotas de uma peça no sistema de manufatura. O grafo de precedências, embora, de menor representatividade, é fundamental na geração das demais representações, uma vez que cada representação utiliza a anterior como input. O segundo modelo representa a flexibilidade de sequiência de uma peça. A mais detalhada e completa representação é a terceira, capacitando a enumeração de todas as possíveis rotas de uma peça em um sistema de manufatura.

Neste artigo, ainda, será abordada a relação entre a representatividade e complexidade computacional para cada nível, auxiliando o processo de seleção do nível de representação mais adequado a um determinado contexto. Embora a descrição de cada representação possa oferecer recomendações sobre tal decisão, a escolha final deverá estar de acordo com os objetivos de cada aplicação.

\subsection{Grafo de precedências}

Precedências são resultantes de restrições mecânicas ou geométricas, que impedem que uma determinada operação seja realizada em uma peça. Restrições geométricas referem-se ao fato de que algumas operações precisam ser executadas antes de outras. Uma operação é considerada geometricamente possível, se o caminho da ferramenta está livre de colisão com a superfície de operações anteriormente executadas. Uma restrição mecânica refere-se ao esforço mecânico que impede a realização de uma operação. Para simplificar este trabalho, restrições geométricas e mecânicas estão implícitas no grafo de precedências.

Quando consideramos a flexibilidade de manufaturas, podemos representar os processos por grafos direcionados (ou dígrafos) (Borenstein, 2000; Hutchinson e Pflughoeft, 1994), nos quais os nós representam as operações, e os arcos estão associados às precedências existentes entre estas operações. Se existe um arco de uma operação $o_{i}$ para uma operação $o_{j}$, significa que a operação $o_{j}$ só pode ser executada se a operação $o_{i}$ já foi realizada. Este grafo pode ser formalmente definido como segue:

Definição 1: $O$ grafo de precedências é um dígrafo $(O, P)$, no qual $O=\left\{o_{1}, o_{2}, \ldots, o_{n}\right\}$ é o conjunto de operações e $P$ é $o$ conjunto de precedências definido como

$$
P=\left\{\left(o_{i}, o_{j}\right) \in O \times O \mid o_{i} \text { precede } o_{j}\right\}
$$

Este grafo pode ser representado por uma matriz $n \times \mathrm{X} n$ de valores binários, em que $\mathbf{A}_{i j}$ é igual a $1 \mathrm{se}$, e apenas se, $\left(o_{i}, o_{j}\right)$ $\in P$. A Tabela 1 apresenta as características de operações de uma peça, enquanto a Figura 1 mostra o grafo de precedências e a matriz adjacente correspondentes.

A geração deste grafo pode ser conduzida usando-se um sistema interativo, no qual o engenheiro de manufatura deve definir as especificações da peça, em termos de precedência de operações e capacidade das máquinas de executá-las. Ba- sicamente, deve-se ao menos prover as informações constantes na Tabela 1.

\subsection{Estados e seqüências de manufatura}

Um estado de manufatura pode ser representado pelas operações já executadas em uma peça durante o processo de produção. Deste modo, um estado de manufatura pode ser representado por um vetor binário de $n$-dimensões

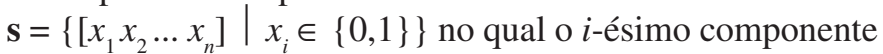
$x_{i}$ é 1 (verdadeiro), se a $i$-ésima operação foi realizada neste estado, e 0 (falso), caso contrário. Esta representação é computacionalmente muito apropriada, uma vez que apresenta o mesmo formato da matriz de precedência. Por exemplo, o estado inicial da peça descrita na Tabela 1 pode ser representado por um vetor binário de quatro dimensões [0000] (correspondente a manufatura no estágio 0 de uma peça). Se a primeira operação no processo de manufatura é a operação 1 , o segundo estado do processo pode ser representado pelo vetor [1000]. Uma vez definido o estado de manufatura, podemos representar uma sequiência de manufatura por uma lista ordenada de $n+1$ estados de manufatura, $\mathbf{s}_{0}, \mathbf{s}_{1}, \ldots, \mathbf{s}_{n}$. (por exemplo, [0000], [0100], [1100], [1101], [1111]).

Nem todos os vetores $n$-dimensionais podem caracterizar um estado. Por exemplo, para a peça especificada na Tabela 1 , o vetor binário [1001] não corresponde a um possível estado de manufatura, pois não respeita as precedências definidas no grafo de precedências. Conseqüentemente, nem todas as sequiências de manufatura serão válidas dentro de um sistema. O conjunto de todos os possíveis estados de manufatura para uma determinada peça será chamado de $F S$. Deste modo, para uma sequiência de manufatura $\mathbf{s}_{0}, \mathbf{s}_{1}, \ldots, \mathbf{s}_{n}$ ser viável, ela precisa obedecer às seguintes propriedades: (i) todas as operações do estado $\mathbf{s}_{i}$ devem estar presentes no estado $\mathbf{s}_{i+1}$; (ii) existe exatamente uma operação no estado $\mathbf{s}_{i+1}$ que não está no estado $\mathbf{s}_{i}$; e (iii) $\mathbf{s}_{i} \in F S, \forall i$.

Tabela 1. Especificação das operações a serem executadas na peça 1.

\begin{tabular}{ccc}
\hline Operação & Precedência & $\begin{array}{c}\text { Máquinas (tempo de operação em } \\
\text { minutos) }\end{array}$ \\
\hline 1 & - & M1 (20) ou M2 (25) \\
2 & - & M2 (30) ou M3 (35) \\
3 & - & M1 (15) \\
4 & 1,2 & M4 (20) \\
\hline
\end{tabular}

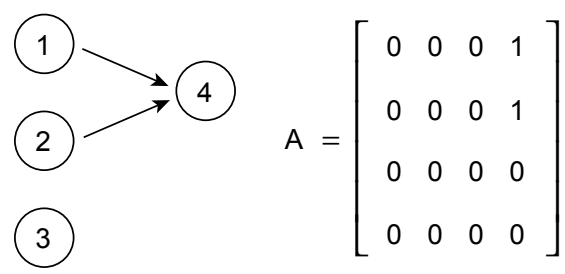

Figura 1. Grafo de precedências e matriz adjacente. 
Gerar todas as possíveis seqüências de manufatura, significa representar e conectar ordenadamente todos os estados que constituem as diferentes sequiências. Uma vez que diferentes seqüências podem compartilhar os mesmos estados de manufatura, é possível criar uma representação que tire vantagem desta propriedade. A próxima seção discute tal representação.

\subsection{Grafo de transição de estados para fle- xibilidade de seqüência}

Fornecido o grafo de precedências $(O, P)$, é possível representar todas as sequiências de manufatura usando-se um dígrafo. Os nós no dígrafo correspondem aos possíveis estados de manufatura. Os arcos correspondem às operações de manufatura, ou seja, a transição entre dois estados. Considerando a descrição dos estados mencionada na seção anterior, um grafo de transição de estados para flexibilidade de seqüência pode ser formalmente definido como:

Definição 2: $O$ grafo de transição de estados para flexibilidade de seqüencia é um grafo direcionado (FS, TS), no qual FS é o conjunto de possíveis estados e

$T S=\left\{\left(\mathbf{s}_{1}, \mathbf{s}_{2}\right) \in F S \times F S \mid\left[\mathbf{s}_{2}-\mathbf{s}_{1} \geq \mathbf{0}\right] \wedge\left[\operatorname{sum}\left(\mathbf{s}_{2}\right)-\operatorname{sum}\left(\mathbf{s}_{1}\right)=1\right]\right\}$

é o conjunto de transições entre os estados, onde:

$$
\operatorname{sum}(\mathbf{s})=\sum_{i=1}^{n} x_{i}
$$

A Figura 2 mostra o grafo de transição de estados para flexibilidade de seqüência correspondente ao grafo de precedências na Figura 1.

O número de nós $(|F S|)$ nesta representação é dado por $2^{\mathrm{n}}$ (uma vez que neste caso, a representação do espaço de estados pode ser representada convenientemente como um diagrama de Hasse (Carre, 1979)). O número máximo de arcos no grafo pode ser computado como segue:

$$
|T S|=\sum_{i=1}^{n} i \times\left(\begin{array}{l}
n \\
i
\end{array}\right)
$$

$\mathrm{O}$ aspecto útil desta representação é que ela pode ser obtida diretamente da matriz adjacente do grafo de precedências, tornando-se bastante conveniente para implementação computacional. A Figura 3 mostra o algoritmo que gera o grafo de transição de estados para flexibilidade de sequiência. Tal algoritmo possui três inputs: a matriz adjacente do grafo de precedências de uma peça, $\mathbf{A}$, um nodo pertencente ao grafo de transição de estados que está sendo gerado, cs, e o próprio grafo de transição de estados para flexibilidade de sequiência, $S T G$. Na primeira iteração do algoritmo, cs é o estado inicial de manufatura definido como [00...0] e $S T G$ é o grafo $(F S, T S)$, tal que $F S=\{\mathbf{c s}\}$ e $T S=\{\varnothing\}$.

\subsection{Grafo de transição de estados para fle- xibilidade de roteamento}

Para representar a flexibilidade de roteamento, é necessário incorporar as máquinas requeridas na execução de cada operação. Assim, um estado de manufatura abrangendo a

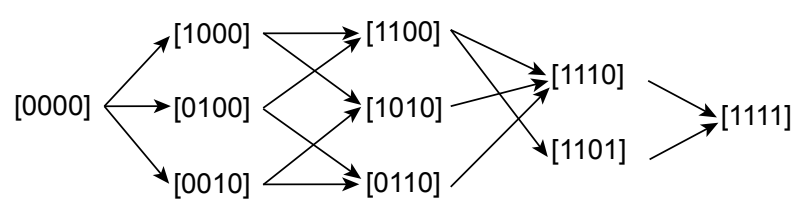

\section{Figura 2. Grafo de transição de estados para} flexibilidade de seqüiência.

flexibilidade de roteamento pode ser representado por um vetor bidimensional $\mathbf{s r}=(\mathbf{s}, \mathbf{m})$, em que $\mathbf{s} \in F S$, e $\mathbf{m}$ é um vetor $n$-dimensional tal que $\mathbf{m}=\left(m_{1}, m_{2}, \ldots, m_{n}\right)$, no qual o $i$-ésimo componente $m_{i}$ assume o valor da máquina na qual a operação $o_{i}$ foi executada. Se o valor do componente $x_{i}$ no vetor $\mathbf{s}$ é zero, então $m_{i}$ também será igual a zero. Por exemplo, o estado ([1110], (M1,M2,M1,0)) indica que as operações 1,2 e 3 já foram executadas utilizando as máquinas M1, M2 e M1, respectivamente. Obviamente, só podemos associar em um estado uma máquina capaz de executar uma determinada operação, gerando a seguinte condição: se $o_{i} \in$ $O$, então pode-se caracterizar um estado de manufatura, se, e somente se, $m_{i} \in M\left(o_{i}\right)$, em que $M\left(o_{i}\right)=\{m \in M \mid m$ é capaz de executar a operação $\left.o_{i}\right\}$. O conjunto de todos os possíveis estados de manufatura, considerando a flexibilidade de roteamento será denominado FSR.

Definimos o predicado $c p_{i}(x, \mathbf{x})$ para determinar se $x$ é ou não o $i$-ésimo componente do vetor $\mathbf{x}$. Por exemplo, para o vetor $\mathbf{x}=(1,7,3,5), c p_{2}(7, \mathbf{x})=V($ verdadeiro $)$, e $c p_{1}(3, \mathbf{x})=F($ falso).

Note que um estado de manufatura $\mathbf{s r} \in F S R$ pode ser obtido de um estado de manufatura $\mathbf{s} \in F S$ pela atribuição de máquinas a cada operação. Dependendo da flexibilidade de máquinas de um sistema de manufatura, um estado $\mathbf{s}$ pode estar relacionado com diferentes estados sr. Por outro lado, cada estado sr está associado a apenas um estado $\mathbf{s}$. Sendo assim, uma rota pode ser definida como uma lista ordenada de $n+1$ estados de manufatura, $\mathbf{s r}_{0}, \mathbf{s r}_{1}, \ldots, \mathbf{s r}_{n}$, tal que $\mathbf{s r}$ $\in F S R, \forall i$. Uma rota $\mathbf{s r}_{0}, \mathbf{s r}_{1}, \ldots, \mathbf{s r}_{\mathrm{n}}$ será considerada viável se: (i) a correspondente seqüência de manufatura $\mathbf{s}_{0}, \mathbf{s}_{1}, \ldots$, $\mathbf{s}_{n}$ também for viável; e (ii) $c p_{1}\left(\mathbf{s}_{i}, \mathbf{s r}_{i}\right)=V, \forall i$. Um exemplo de uma rota para a peça definida na Tabela 1 é: ([0000], $(0,0,0,0)),([0100],(0, \mathrm{M} 2,0,0)),([1100], \quad(\mathrm{M} 2, \mathrm{M} 2,0,0))$, ([1101], (M2,M2,0,M4)), ([1111], (M2,M2,M1,M4)).

Como diferentes rotas compartilham estados de manufatura comuns, é possível representar o conjunto de todos os roteamentos utilizando uma representação em dígrafo, em que os nós representam os estados da manufatura, e os arcos representam as transições entre estes estados. O grafo de transição de estados para flexibilidade de roteamento pode ser formalmente definido como:

Definição 3: $O$ grafo de transição de estados para flexibilidade de roteamento é um dígrafo (FSR, TSR), no qual FSR é o conjunto de possíveis estados de manufatura e

$T S R=\left\{\left(\mathbf{s r}_{1}, \mathbf{s r}_{2}\right) \in F S R \times F S R \mid\left[c p_{1}\left(\mathbf{s}_{1}, \mathbf{s r}_{1}\right)\right] \wedge\left[c p_{1}\right.\right.$ $\left.\left.\left(\mathbf{s}_{2}, \mathbf{s r}_{2}\right)\right] \wedge\left[\left(\mathbf{s}_{1}, \mathbf{s}_{2}\right) \in T S\right]\right\}$ 


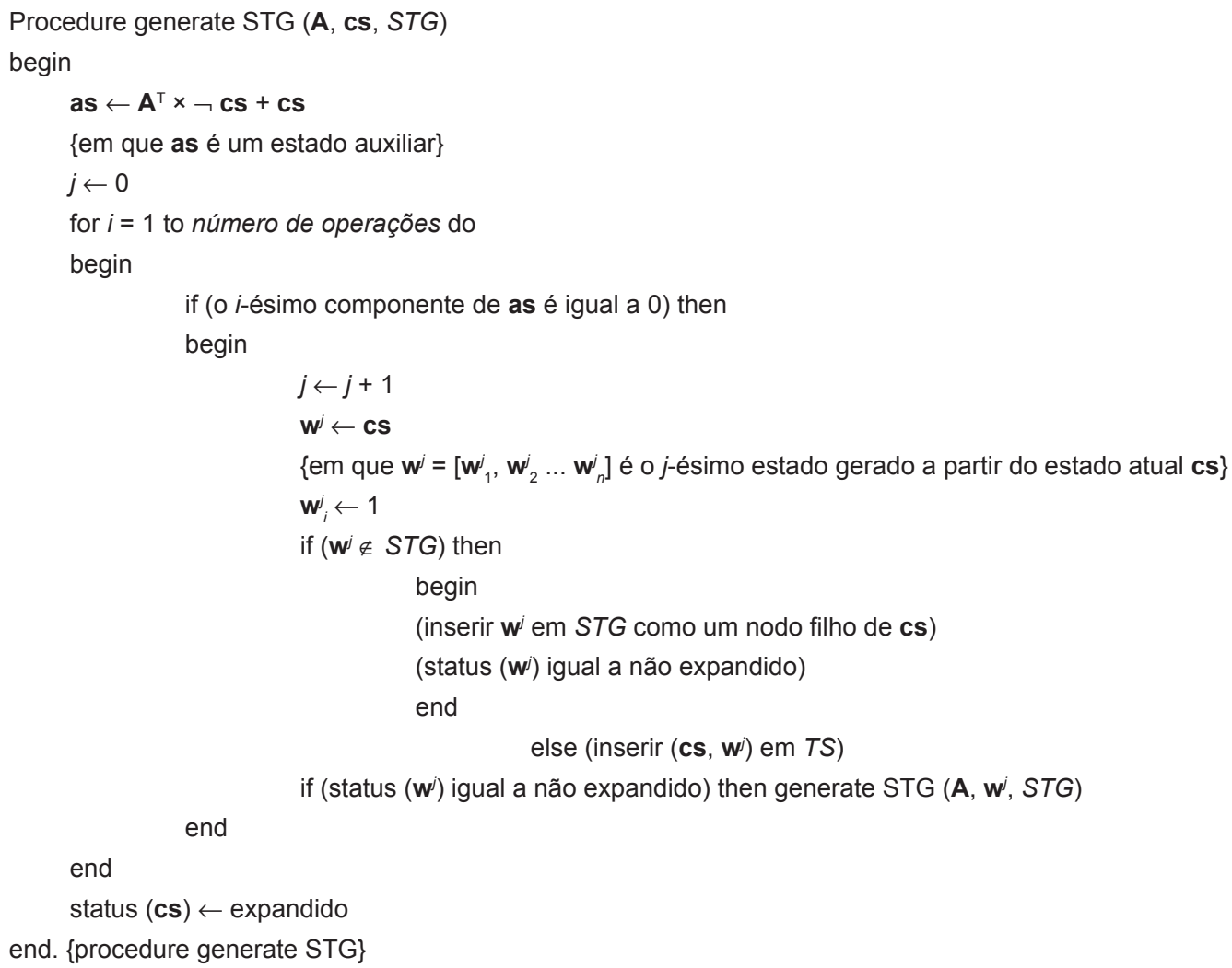

Figura 3. Algoritmo Generate STG.

\section{é o conjunto de transições entre os estados.}

A Figura 4 apresenta o grafo de transição de estados para flexibilidade de roteamento correspondente ao grafo de precedências da Figura 1 e às informações de máquinas existentes na Tabela 1.

As possíveis rotas correspondem aos caminhos no grafo $(F S R, T S R)$, começando pelo estado inicial representado pelo vetor $([00 \ldots 0],(0,0, \ldots, 0))$ até o estado final representado pelo vetor $\left([11 \ldots 1],\left(m_{1}, m_{2}, \ldots, m_{n}\right)\right)$, em que $m_{i} \neq 0, \forall i$. O grafo de transição de estados para flexibilidade de roteamento pode ser facilmente obtido expandindo-se o grafo de transição de estados para flexibilidade de sequiência. $\mathrm{O}$ número de nós $(|F S R|)$ e arcos (|TSR|) nesta representação são, contudo, elevados. Numa situação de total flexibilidade de seqüência e máquinas (100\% de flexibilidade de roteamento), o número de nós e arcos é fornecido pelas equações:

$$
\begin{aligned}
& \left.R\left|=1+\sum_{i=1}^{n}\left(\begin{array}{l}
n \\
i
\end{array}\right) \times\right| m\right|^{i} \\
& \left.R\left|=\sum_{i=1}^{n} \frac{n !}{(n-i) !} \times\right| m\right|^{i}
\end{aligned}
$$

em que $m$ é número de máquinas no sistema de manufatura. Ambas as equações representam a maior complexidade (caso mais extremo). Todavia, na prática, o número de nós e arcos é consideravelmente menor. Testes realizados demonstram que o limite prático computacional da representação ocorre quando se tem 6 operações e 6 máquinas capazes de operarem as seis operações (total flexibilidade de máquina e total flexibilidade de seqüência), ou seja, não existe nenhuma precedência entre as operações. A partir desta situação, a representação ocupa um espaço de memória, em muito, superior à disponível pelos atuais computadores. Porém, é possível, por meio de artifícios de modelagem, tais como a agregação de máquinas e uso de heurísticas para prunar a geração completa do grafo, simplificar situações de alta flexibilidade, possibilitando a sua representação por meio do grafo de transição de estados. A próxima seção ilustra possíveis aplicações das representações desenvolvidas.

\section{Exemplos}

Esta seção pretende demonstrar o uso das representações desenvolvidas em problemas diretamente relacionados com o roteamento na manufatura, incluindo sua mensuração. $\mathrm{O}$ objetivo neste estudo de caso é mostrar como a representação da flexibilidade de roteamento pode ser aplicada, para resolver problemas na seleção do melhor roteamento de um conjunto de peças em um sistema de manufatura e, assim, encontrar o melhor balanço entre produção e minimização dos custos. Este problema já foi estudado por Chandra e Tombak (1992), Chen e Chung (1996), e Das e Nagendra (1997). Entretanto, todos os modelos desenvolvidos por estes autores assumem que as possíveis rotas de manufatura são previamente geradas, sem mencionar detalhes de como elas foram obtidas, dadas as características do sistema de 


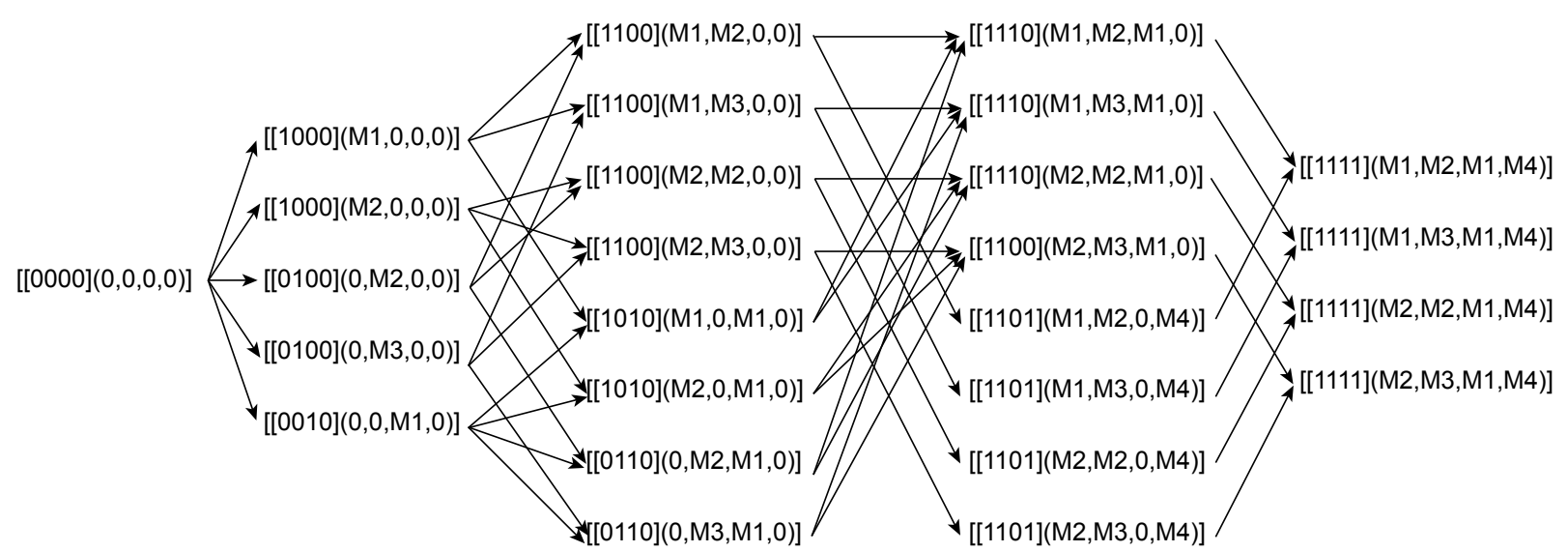

Figura 4. Grafo de transição de estados para flexibilidade de roteamento - Peça 1.

manufatura. Este trabalho contribui para a solução deste problema, por meio da integração do planejamento dos planos de manufatura com a seleção das possíveis rotas para ele.

Para selecionar o melhor roteamento de manufatura para um conjunto de peças, é necessário gerar todas as possíveis soluções, isto é, todas possíveis rotas de uma peça no sistema de manufatura. Uma vez que os roteamentos estão associados a caminhos no grafo, a seleção do melhor roteamento pode ser entendida como um problema de busca. Para isso, é necessário definir um critério que compare os diferentes planos de manufatura. Um critério bem aceito é atribuir um custo a cada caminho do grafo, por meio da soma dos custos individuais de cada arco (que representam as dificuldades de suas operações). Uma grande variedade de fatores pode ser considerada na atribuição dos custos dos arcos, incluindo o tempo de duração do setup, disponibilidade dos recursos, confiabilidade, entre outros. A união destes fatores pode ser expressa como a complexidade da operação (Borenstein, 2000). A atribuição da complexidade da operação durante o projeto dos estágios é altamente dependente da experiência do engenheiro responsável pelo sistema de manufatura.

Este problema pode ser formulado como um modelo de programação linear inteira (PLI). O objetivo deste modelo é levar em consideração as características do sistema de manufatura, e tentar identificar o melhor conjunto de rotas que minimizam a complexidade total das operações. O seguinte modelo, bem aceito na literatura (Chandra e Tombak, 1992; Chen e Chung, 1996), pode ser formulado.

$$
\mathrm{n} \sum_{i} \sum_{h} c_{i h} x_{i h}
$$

$$
\begin{array}{ll}
\sum_{h b_{i l h}=1} \frac{t_{i l}}{p_{l}} x_{i h} \leq T_{l} & \forall l \\
x_{i h} \geq d_{i} & \forall i \\
\geq 0 & \forall i, h
\end{array}
$$

Em que: $i$ é o índice do tipo de peça; $i=1,2, \ldots, n$;

$l$ é o índice do tipo de máquina; $l=1,2, \ldots . m$;

$h$ é o índice de um caminho no grafo de transição de estados para flexibilidade de roteamento; $h=1,2, \ldots, H$;

$t_{i l}$ é o tempo requerido para processar uma unidade da peça $i$ na máquina $l$;

$T_{l}$ é o tempo disponível para processamento na máquina $l$;

$p_{l}$ é a probabilidade da máquina $l$ estar operando em um determinado momento;

$b_{i l h}$ é um parâmetro zero-um, em que um indica que o produto $i$ pode ser produzido na máquina $l$ pelo caminho $h$;

$c_{i h}$ é a complexidade da operação de uma peça $i$ usando um caminho $h$;

$d_{i}$ é a demanda mínima que deve ser satisfeita pela peça $i$; e

$x_{i h}$ é o fluxo da peça $i$ pelo caminho $h$;

A restrição (1) limita o tempo de processamento em cada máquina, considerando sua confiabilidade. A demanda mínima de cada peça é garantida pela restrição (2). Deve-se notar que este modelo baseia-se nos seguintes pressupostos (que simplificam sua estrutura, concentrando os efeitos da flexibilidade de roteamento na performance do sistema de manufatura): (i) o tempo de setup das máquinas está incluído nos tempos de processamento das operações; e (ii) a existência de filas não está sendo levada em consideração. Este modelo apresenta um número de variáveis igual a $\sum^{n} H_{i}$, em que $H_{i}$ é o numero de rotas da peça $i$. Desta forma, ${ }^{i}$ esta formulação é limitada pela capacidade de geração do grafo de transição de estados de uma peça. Porém, se considerarmos que a geração de rotas pode acontecer sem a necessidade de armazenar todo o grafo, utilizando-se algoritmos apropriados, esta limitação pode ser facilmente contornada, restringindo sua solução à capacidade do programa de solução do PLI.

Suponha que as peças apresentadas nas Tabelas 1 (Peça 1) e 2 (Peça 2) são manufaturadas no sistema descrito na Ta- 
Tabela 2. Especificação das operações a serem executadas na peça 2.

\begin{tabular}{ccc}
\hline Operação & Precedência & $\begin{array}{c}\text { Máquinas (tempo de operação } \\
\text { em minutos) }\end{array}$ \\
\hline 1 & - & M2 (15) ou M3 (20) \\
2 & 1 & M1(20) \\
3 & - & M1 (10) ou M2 (15) \\
4 & 3 & M3 (20) \\
\hline
\end{tabular}

bela 3 (veja Figura 5). O conveyor pode mover-se em apenas uma direção com velocidade de $0,5 \mathrm{~m} / \mathrm{s}$. A distância em metros entre as cinco estações de trabalho, considerando a trajetória do conveyor é mostrada na Tabela 3 , em que I e O (input e output) representam a entrada e saída da célula de produção, respectivamente. Esta tabela também mostra a confiabilidade de cada máquina. Arbitra-se $T_{l}=360000 \forall l$, $d_{1}=1000$ unidades, e $d_{2}=800$ unidades.

As Tabelas 4 e 5 mostram possíveis pesos atribuídos a cada rota no grafo de transição de estados para flexibilidade de roteamento das Peças 1 e 2. Estes pesos foram computados somando-se dois fatores. O primeiro fator está associado ao tempo de operação (em minutos), representando as necessidades de manipulação, posicionamento e configuração para executar uma operação em uma determinada máquina. $\mathrm{O}$ segundo fator leva em consideração o custo de transporte (em minutos).

Este sistema gerou uma solução ótima de 146.222,3, com 930 e 70 unidades da Peça 1 sendo processadas nas rotas $(2,2)-(3,1)-(1,2)-(4,4)$ e $(3,1)-(1,1)-(2,3)-(4,4)$, respectivamente, e 380 e 420 unidades da Peça 2 produzidas nas rotas $(1,3)-(2,1)-(3,1)-(4,3)$ e $(3,1)-(1,2)-(4,3)-(2,1)$, respectivamente. Para atender às demandas mínimas, as máquinas M1 e M2 tornaram-se gargalos, com suas capacidades totalmente utilizadas. Conseqüentemente, o resultado encontrado no modelo de programação inteira utiliza rotas que minimizam os custos de processamento nestas duas máquinas, em detrimento da seleção de rotas com uma complexidade menor. Todas as rotas selecionadas têm, relativamente, altos custos de complexidade em uma análise global (veja Tabela 4 e Tabela 5). A Tabela 6 mostra como as rotas podem mudar com a alteração na demanda das Peças, e como as rotas de menor complexidade são relegadas conforme as máquinas tornam-se sobrecarregadas. Sendo, assim, quando não existir nenhum gargalo no sistema, apenas uma rota será selecionada para cada peça. Neste caso, a flexibilidade de roteamento torna-se desnecessária. Embora com alguma perda de eficiência, a flexibilidade de roteamento tornou possível, ao sistema de manufatura, atender às demandas solicitadas.

Também é possível utilizar o grafo de transição de estados para computar a flexibilidade de roteamento de um sistema, para o processamento de um produto ou uma combinação de produtos. A literatura descreve diversos métodos de mensuração para a flexibilidade de roteamento (Chen e Chung, 1996, Sarker, Krishnamurthy e Kuthethur, 1994; Shewchuk, 1999). A mensuração adequada deve incluir os seguintes aspectos: (i) o número de roteamentos disponíveis; (ii) a efici-
Tabela 3. Distâncias (em metros) entre as máquinas.

\begin{tabular}{cccccccc}
\hline Máquina & I & O & M1 & M2 & M3 & M4 & Confiabilidade \\
\hline I & 0 & 7 & 2 & 12 & 5 & 9 & - \\
O & 8 & 0 & 10 & 5 & 12 & 2 & - \\
M1 & 12 & 5 & 0 & 10 & 3 & 7 & 0,90 \\
M2 & 2 & 9 & 4 & 0 & 7 & 11 & 0,95 \\
M3 & 9 & 2 & 11 & 7 & 0 & 4 & 0,85 \\
M4 & 5 & 12 & 7 & 3 & 10 & 0 & 0,90 \\
\hline
\end{tabular}

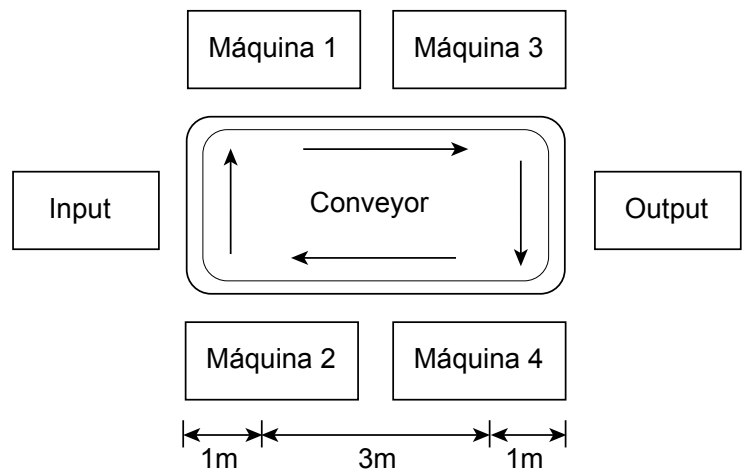

Figura 5. Layout do sistema de manufatura.

ência de cada rota; e (iii) a disponibilidade de utilização das rotas. O grafo de transição de estados para flexibilidade de roteamento é capaz de capturar todos estes aspectos, permitindo mensurar a flexibilidade de roteamento de diversas formas. Para ilustrar o potencial da representação desenvolvida, utilizamos uma mensuração baseada na abordagem analítica de Kochikar e Narendram (1992) para calcular a flexibilidade do sistema de manufatura. A seguinte notação (similar à apresentada em Kochikar e Narendram (1992)) é usada para mensurar a flexibilidade de roteamento.

- l é o número de máquinas em funcionamento;

- $i$ é o número de peças em processamento. A $k$-ésima operação na peça $i$ é denotada pelo par ordenado $(i, k)$;

- $j$ é o índice para os estágios de processamento $(j=0$, significa que nenhuma operação foi realizada na peça). Um estágio de processamento inclui o transporte da peça até uma máquina, a execução da operação e sua saída da máquina;

- $v_{i}$ é a fração da demanda total representada pela peça $i$;

$$
\sum_{i \in W^{s}} v_{i}=1
$$

- $W^{S}$ é o conjunto de peças sendo processadas no sistema;

- $\Omega_{\mathrm{i}}=\left\{k \mid 1 \leq k \leq n_{i}\right\}$ é o índice de todas as operações necessárias na peça $i$;

- $L_{i k}$ é o conjunto de máquinas alternativas que podem executar a operação $(i, k)$;

- $t^{c}{ }_{l i k}$ é o tempo necessário para a operação $(i, k)$ ser efetuada na máquina $l$, definido como:

$$
t_{l i k}^{c}=\left(t_{s}+t_{l u}+t_{t x}+t_{m}\right)_{l i k}
$$


Tabela 4. Enumeração das rotas para peça 1.

\begin{tabular}{|c|c|c|c|c|}
\hline Número & Rotas & $\begin{array}{c}\text { Custo de } \\
\text { processamento }\end{array}$ & $\begin{array}{c}\text { Custo de } \\
\text { transporte }\end{array}$ & $\begin{array}{c}\text { Complexidade } \\
\text { operacional }\end{array}$ \\
\hline 1 & $(1,1)-(2,3)-(3,1)-(4,4)$ & 90 & 1,17 & 91,17 \\
\hline 2 & $(1,1)-(2,3)-(4,4)-(3,1)$ & 90 & 0,70 & 90,70 \\
\hline 3 & $(1,1)-(3,1)-(2,3)-(4,4)$ & 90 & 0,70 & 90,70 \\
\hline 4 & $(1,1)-(3,1)-(2,2)-(4,4)$ & 85 & 1,13 & 86,13 \\
\hline 5 & $(1,1)-(2,2)-(3,1)-(4,4)$ & 85 & 1,17 & 86,17 \\
\hline 6 & $(1,1)-(2,2)-(4,4)-(3,1)$ & 85 & 1,23 & 86,23 \\
\hline 7 & $(1,2)-(2,3)-(3,1)-(4,4)$ & 95 & 1,63 & 96,63 \\
\hline 8 & $(1,2)-(2,3)-(4,4)-(3,1)$ & 95 & 1,17 & 96,17 \\
\hline 9 & $(1,2)-(2,2)-(3,1)-(4,4)$ & 90 & 1,17 & 91,17 \\
\hline 10 & $(1,2)-(2,2)-(4,4)-(3,1)$ & 90 & 1,17 & 91,17 \\
\hline 11 & $(1,2)-(3,1)-(2,3)-(4,4)$ & 95 & 1,43 & 96,43 \\
\hline 12 & $(1,2)-(3,1)-(2,2)-(4,4)$ & 90 & 1,63 & 91,63 \\
\hline 13 & $(2,3)-(1,1)-(3,1)-(4,4)$ & 90 & 1,17 & 91,17 \\
\hline 14 & $(2,3)-(1,1)-(4,4)-(3,1)$ & 90 & 1,17 & 91,17 \\
\hline 15 & $(2,3)-(1,2)-(3,1)-(4,4)$ & 95 & 1,17 & 96,17 \\
\hline 16 & $(2,3)-(1,2)-(4,4)-(3,1)$ & 95 & 1,17 & 96,17 \\
\hline 17 & $(2,3)-(3,1)-(1,1)-(4,4)$ & 90 & 1,17 & 91,17 \\
\hline 18 & $(2,3)-(3,1)-(1,2)-(4,4)$ & 95 & 1,33 & 96,33 \\
\hline 19 & $(2,2)-(1,1)-(4,4)-(3,1)$ & 85 & 1,17 & 86,17 \\
\hline 20 & $(2,2)-(1,1)-(3,1)-(4,4)$ & 85 & 1,17 & 86,17 \\
\hline 21 & $(2,2)-(1,2)-(3,1)-(4,4)$ & 90 & 1,17 & 91,17 \\
\hline 22 & $(2,2)-(1,2)-(4,4)-(3,1)$ & 90 & 1,17 & 91,17 \\
\hline 23 & $(2,2)-(3,1)-(1,1)-(4,4)$ & 85 & 1,17 & 86,17 \\
\hline 24 & $(2,2)-(3,1)-(1,2)-(4,4)$ & 90 & 1,63 & 91,63 \\
\hline 25 & $(3,1)-(1,1)-(2,3)-(4,4)$ & 90 & 0,70 & 90,70 \\
\hline 26 & $(3,1)-(1,1)-(2,2)-(4,4)$ & 85 & 1,17 & 86,17 \\
\hline 27 & $(3,1)-(1,2)-(2,3)-(4,4)$ & 95 & 1,17 & 96,17 \\
\hline 28 & $(3,1)-(1,2)-(2,2)-(4,4)$ & 90 & 1,17 & 91,17 \\
\hline 29 & $(3,1)-(2,3)-(1,1)-(4,4)$ & 90 & 1,17 & 91,17 \\
\hline 30 & $(3,1)-(2,3)-(1,2)-(4,4)$ & 95 & 1,17 & 96,17 \\
\hline 31 & $(3,1)-(2,2)-(1,1)-(4,4)$ & 85 & 1,17 & 86,17 \\
\hline 32 & $(3,1)-(2,2)-(1,2)-(4,4)$ & 90 & 1,17 & 91,17 \\
\hline
\end{tabular}

em que $t_{s}=$ tempo de setup; $t_{l u}=$ tempo de processamento; $t_{t x}$ $=$ tempo de troca de ferramenta; e $t_{m}=$ tempo na máquina;

- $s_{i j}$ é a variável que indica o conjunto de estados em que a peça $i$ pode estar após completar o estágio $j$ de processamento;

- $\xi\left(s_{i j}\right)$ denota a cardinalidade de $s_{i j}$;

- $r_{l i k}$ é o fator de eficiência que expressa o sucesso de uma operação ser realizada, usando uma determinada máquina:

$$
r_{l i k}=r_{l}^{a} / r_{l i k}^{b}
$$

em que $r_{l}^{a}$ é a disponibilidade (proporcional ou única) da máquina $l$; e $r^{b}{ }_{l i k}$ é o tempo de operação normalizado, dado por:

$$
r^{b}{ }_{l i k}=\frac{t_{l i k}}{\min _{l^{*} \in L_{i k}}\left[t^{c}{ }_{l^{*} i k}\right]}
$$

A flexibilidade de roteamento da peça $i$, considerando-se um conjunto de máquinas $M$, é dada pela seguinte mensuração:

$$
r f_{i}(M)=1 / n_{i} \sum_{j=0}^{n_{i}-1} \frac{\sum_{\xi\left(s_{i j}\right)} \sum_{k \in \Omega \Omega_{i, s i j}^{A}} \sum_{i \in M, k \in L_{i j}} r_{i k}}{\frac{n_{i} !}{\left(n_{i}-j+1\right) !} \times|M|^{j+1}}
$$

em que $n_{i}$ é o número de operações requeridas pela peça $i ; \Omega^{A} i, s_{i j} \subseteq \Omega i$ é o conjunto de operações que podem ser executadas na peça $i$ no estágio $j$, levando-se em consideração o estado $s_{i j}$ de processamento e $r f_{i} \in[0,1]$. A flexibilidade de roteamento da peça $r f_{i}$ é a razão entre o número de rotas disponíveis e o total de rotas existentes (total flexibilidade) em cada estágio de processamento. A mensuração calculada para uma determinada peça pode ser usada para verificar os 
Tabela 5. Enumeração das rotas para peça 2

\begin{tabular}{ccccc}
\hline Número & Rotas & $\begin{array}{c}\text { Custo de } \\
\text { processamento }\end{array}$ & $\begin{array}{c}\text { Custo de } \\
\text { Transporte }\end{array}$ & $\begin{array}{c}\text { Complexidade } \\
\text { operacional }\end{array}$ \\
\hline 1 & $(1,2)-(2,1)-(3,1)-(4,3)$ & 65 & 0,70 & 65,70 \\
2 & $(1,2)-(2,1)-(3,2)-(4,3)$ & 70 & 1,17 & 71,17 \\
3 & $(1,3)-(2,1)-(3,1)-(4,3)$ & 70 & 0,70 & 70,70 \\
4 & $(1,3)-(2,1)-(3,2)-(4,3)$ & 75 & 1,17 & 76,17 \\
5 & $(1,2)-(3,1)-(2,1)-(4,3)$ & 65 & 0,70 & 65,70 \\
6 & $(1,2)-(3,2)-(2,1)-(4,3)$ & 70 & 0,70 & 70,70 \\
7 & $(1,3)-(3,1)-(2,1)-(4,3)$ & 70 & 0,70 & 70,70 \\
8 & $(1,3)-(3,2)-(2,1)-(4,3)$ & 75 & 0,70 & 75,70 \\
9 & $(1,2)-(3,1)-(4,3)-(2,1)$ & 65 & 1,17 & 66,17 \\
10 & $(1,2)-(3,2)-(4,3)-(2,1)$ & 70 & 1,17 & 71,17 \\
11 & $(1,3)-(3,1)-(4,3)-(2,1)$ & 70 & 1,17 & 71,17 \\
12 & $(3,1)-(1,2)-(4,3)-(2,1)$ & 65 & 1,17 & 66,17 \\
13 & $(3,1)-(1,3)-(4,3)-(2,1)$ & 70 & 0,70 & 70,70 \\
14 & $(3,2)-(1,2)-(4,3)-(2,1)$ & 70 & 1,17 & 71,17 \\
15 & $(3,2)-(1,3)-(4,3)-(2,1)$ & 75 & 1,17 & 76,17 \\
16 & $(3,1)-(1,2)-(2,1)-(4,3)$ & 65 & 0,70 & 65,70 \\
17 & $(3,1)-(1,3)-(2,1)-(4,3)$ & 70 & 0,70 & 70,70 \\
18 & $(3,2)-(1,2)-(2,1)-(4,3)$ & 70 & 0,70 & 70,70 \\
19 & $(3,2)-(1,3)-(2,1)-(4,3)$ & 75 & 1,17 & 76,17 \\
20 & $(3,1)-(4,3)-(1,2)-(2,1)$ & 65 & 0,70 & 65,70 \\
21 & $(3,1)-(4,3)-(1,3)-(2,1)$ & 70 & 0,70 & 70,70 \\
22 & $(3,2)-(4,3)-(1,2)-(2,1)$ & 75 & 1,17 & 76,17 \\
\hline
\end{tabular}

Tabela 6. Enumeração dos roteamentos para peça 2.

\begin{tabular}{c|cc|c}
\hline & \multicolumn{2}{|c|}{$\boldsymbol{x}^{*}{ }_{i h}-$ Número do Roteamento (Complexidade) } & \\
\hline Demanda (Peça 1, Peça 2) & Roteamento para Peça 1 & Roteamento para Peça 2 & Gargalos \\
\hline$(1000,800)$ & $930-24(91,63), 70-25(90,70)$ & $380-3(70,70), 420-12(66,17)$ & M1, M2 \\
$(800,800)$ & $160-4(86,13), 580-24(91,63), 60-25(90,70)$ & $800-12(66,17)$ & M1, M2 \\
$(600,800)$ & $370-4(86,13), 62-24(91,63), 168-32(91,17)$ & $800-12(66,17)$ & M1, M2 \\
$(600,600)$ & $570-4(86,13), 30-32(91,17)$ & $600-12(66,17)$ & M1 \\
$(600,400)$ & $600-4(86,13)$ & $340-1(65,70), 60-12(66,17)$ & M2 \\
$(400,400)$ & $400-4(86,13)$ & $400-1(65,70)$ & - \\
\hline
\end{tabular}

ganhos em flexibilidade de roteamento, considerando todas a peças sendo processadas no sistema.

$$
R F=\sum_{i \in W^{s}} v_{i} R F_{i}\left(M^{s}\right)
$$

É possível utilizar as equações definidas em (3) e (4) para computar a flexibilidade de processamento para as Peças 1 e 2, e a flexibilidade do sistema global. Os valores encontrados para a flexibilidade de roteamento correspondente às Peças 1 e 2 são 0,087 e 0,0618 , respectivamente, enquanto a flexibilidade de roteamento do sistema global é igual a 0,0759 . Os resultados são coerentes, uma vez que a Peça 1 é levemente mais flexível do que a Peça 2, dado que seu plano de manufatura tem menos precedências entre as operações. Entretanto, tanto as peças como o sistema de manufatura, oferecem um nível bastante reduzido de flexibilidade de roteamento. É importante notar que esta mensuração refere-se a uma potencial flexibilidade de roteamento de uma peça ou sistema de manufatura, uma vez que o número máximo de rotas realmente usado é menor do que o fornecido pelo grafo de transição de estados para flexibilidade de roteamento, como mostrado pelos resultados do exemplo anterior, no qual o número máximo de rotas selecionadas foi 3 para a Peça 1, e 2 para a Peça 2.

Em síntese, com estes exemplos, nossa representação permite que o responsável pela manufatura entenda os efeitos da flexibilidade de roteamento e mensure suas implicações econômicas e operacionais. Além disso, nossa representação fornece uma ferramenta com a qual pode-se continuamente avaliar um sistema sob diferentes condições e critérios. 


\section{Conclusão}

Um método para representar a flexibilidade de roteamento e ser implementado em sistemas flexíveis de manufatura foi desenvolvido. Esta representação permite que a flexibilidade de roteamento seja devidamente explorada, auxiliando, efetivamente, no projeto de sistemas flexíveis de manufatura. Sendo assim, a principal contribuição deste artigo é o desenvolvimento de uma representação compacta para todas as possíveis rotas de uma peça em um sistema de manufatura, utilizando-se informações fornecidas por experts. Tal representação permite um aumento na flexibilidade, capacitando que o sistema escolha o curso de ação mais conveniente de acordo com as condições do sistema de manufatura. A versatilidade desta representação permite que esta seja facilmente vinculada com outras ferramentas de análise e apoio, para o controle e projeto de sistemas flexíveis de manufatura, permitindo que estes sistemas sejam devidamente avaliados, compreendidos e mensurados. O modelo de flexibilidade de roteamento desenvolvido, aliado a modelos analíticos ou de simulação, permitirá predizer o comportamento dinâmico de diferentes sistemas de manufatura, sob variadas condições operacionais. Ele também possibilitará a avaliação da influ- ência da flexibilidade em diversos parâmetros de desempenho, tais como utilização dos recursos, tempo de espera de uma peça, mesmo diante de contingências, como quebra de máquinas ou manutenção.

Uma vez que existe uma necessidade de balancear flexibilidade e custos (Sarker, Krishnamurthy e Kuthethur, 1994), o desenvolvimento de modelos que permitam melhorar a compreensão das consequiências de implementação de manufaturas flexíveis auxilia os gestores na escolha dos níveis de flexibilidade necessários, considerando a menor perda possível de eficiência. Esta é a principal implicação gerencial deste trabalho.

Exemplos foram apresentados evidenciando como a representação pode ser usada para resolver problemas, como os de otimização de roteamentos e mensuração da flexibilidade. Somado a isso, foram demonstradas as limitações computacionais, dando indicadores da melhor representação para cada contexto.

Em resumo, o modelo desenvolvido para a flexibilidade de roteamento fornece uma abordagem inovadora e alternativa, para auxiliar engenheiros e administradores no entendimento da flexibilidade na manufatura, tanto para projeto como controle das operações.

\section{Referências Bibliográficas}

AZZONE, G.; BERTELÈ, U. Techniques for measuring the effectiveness of automation and manufacturing systems. Control and dynamic systems, v. 48, p. 1-45, 1991.

BENJAAFAR, S. Models for performance evaluation of flexibility in manufacturing systems. International journal of production research, v. 32, n. 6, p. 1383-1402, 1994.

BENJAAFAR, S.; RAMAKRISHNAM, R. Modelling, measurement and evaluation of sequence flexibility in manufacturing systems. International journal of production research, v. 34, n. 5, p. 1195-1220, 1996.

BORENSTEIN, D. A directed acyclic graph representation of routing manufacturing flexibility. European journal of operational research, v. 127, n. 1, p. 89-103, 2000.

CARRE, B. Graphs and networks. Oxford: Claredon Press, 1979.

CHANDRA, P.; TOMBAK, M. M. Models for the evaluation of routing and machine flexibility. European journal of operational research, v. 60, p. 156-165, 1992.

CHEN, I. J.; CHUNG, C. H. An examination of flexibility measurements and performance of flexible manufacturing systems. International journal of production research, v. 34, n. 2, p. 379-394, 1996.

DAS, S. K.; NAGENDRA, P. Selection of routes in a flexible manufacturing facility. International journal of produc- tion economics, v. 48, p. 237-247, 1997.

GUPTA, D.; BUZACOTT, J. A. A framework for understanding flexibility of manufacturing systems. Journal of manufacturing systems, v. 8, n. 2, p. 89-97, 1989.

HANCOCK, T. M. Effects of alternates routings under variable lot size conditions. International journal of production research, v. 27, p. 247-259, 1989.

HUTCHINSON, G.K.; PFLUGHOEFT, K.A. Flexible process plans: their value in flexible automation systems. International journal of production research, v. 32, n. 3, p. 707719, 1994.

HWANG, C.; GUO, T. Y; SHIEH, L. S. A canonical state-space representation for SISO systems using multipoint Jordan CFE. Journal of Franklin institute, v. 328, n. 2-3, p. $207-$ 216, 1991.

KOCHIKAR, V.P.; NARENDRAN, T.T.A framework for assessing the flexibility of manufacturing systems. International journal of production research, v. 30, n. 12, p. 2873-2895, 1992.

LIN, G. Y. J.; SOLBERG, J. J. Effectiveness of flexible routing control. International journal of flexible manufacturing system, v. 3, p. 189-211, 1991.

MORGAN, E. T.; RAZOUK, R. R. Interactive state-space analysis of concurrent systems. IEEE transactional sof- 
tware engineering, v. 13, n. 10, p. 1080-1091, 1987.

SARKER, B.; KRISHNAMURTHY, S.; KUTHETHUR, S. A survey and critical review of flexibility measures in manufacturing systems. Production planning and control, v. 5, n. 6, p. 512-523, 1994.

SETHI, A. K.; SETHI, S. P. Flexible in manufacturing: a survey. International journal of flexible manufacturing systems, v. 2, p. 289-328, 1990.

SHEWCHUK, J. P. A set of generic flexibility measures for manufacturing applications. International journal of production research, v. 37, n. 13, p. 3017-3042, 1999.

WEI, Y.; EGBELU, P. J. Process alternative generation from product geometric design data. IIE transactions, v. 32, p. 71-82, 2000.

YANG, Z.; QIAO, L.; JIANG, L. Improving the performances of part dispatching based on multiple process plans using graph theory. International journal of production research, v. 36, n. 7, p. 1987-2003, 1998.

\title{
ROUTING FLEXIBILITY USING STATE SPACE REPRESENTATION
}

\begin{abstract}
This paper describes a state space representation for routing flexibility in manufacturing systems. Routing flexibility is represented on three different levels, as follows: (i) Operations Precedence Graph; (ii) State Transition Graph of Manufacturing Operation Sequences; and (iii) State Transition Graph of Manufacturing Operation Routes. Each representation is able to represent routing flexibility in different degrees of detail. The third and most complete representation can enumerate all the possible manufacturing operation routes for a given part in the manufacturing system. Bounds for the computation of the representation levels are presented to help the user select the most suitable one for a given context. The versatility of this representation allows it to be linked easily with other analytical and support tools for the to design, operation, and control of flexible manufacturing systems, so that manufacturing flexibility can be properly measured, understood, and evaluated. The efficacy of the representation is demonstrated through its application to problems such as job route selection and routing flexibility measurement in manufacturing systems.
\end{abstract}

Keywords: routing flexibility, state space representation, manufacturing routes design. 